\title{
THERMAL-TIDAL INSTABILITY MODEL OF DWARF NOVAE BELOW THE PERIOD GAP:
}

\section{A UNIFICATION THEORY}

\author{
Y. OSAKI \\ Department of Astronomy, School of Science, \\ University of Tokyo, Bunkyo-ku, Tokyo, 113, Japan
}

\begin{abstract}
A unification theory is proposed in which almost all of outburst behavior in dwarf novae may be understood in a unified way within the basic framework of the disk instability model. In particular, a rich variety of outburst behavior of cataclysmic variables below the period gap may be understood by the thermal-tidal instability model in which the coupling of the two intrinsic instabilities in the accretion disk plays a unique role.
\end{abstract}

\section{A unification model of dwarf nova outbursts}

It is fairly widely accepted that the outbursts of dwarf novae (DNe) can basically be explained by the disk instability (DI) model, at least for those above the well-known period gap. In this model, the mass transfer rate from the secondary star is assumed to be constant but accretion from the disk to the central white dwarf is not steady but intermittent because of the intrinsic instability in accretion disks. The instability itself is a kind of thermal instability associated with ionization of hydrogen.

Another intrinsic instability that operates in accretion disks - called the 'tidal instability' - was later discovered by Whitehurst (1988). In this instability the accretion disk is deformed to an eccentric form and its eccentric pattern slowly precesses in the prograde direction in the inertial frame of reference. The so-called 'superhump' phenomenon observed during superoutbursts of SU UMa stars is now believed to be explained by this tidal instability. The tidal instability is found to occur when the disk's outer edge reaches the 3:1 resonance radius (Whitehurst 1988; Hirose \& Osaki 1990; Lubow 1991), a condition that is possible only in binary systems with extremely low mass ratio, $q$, with $q<0.25$, where $q=M_{2} / M_{1}$ and $M_{1}$ and $M_{2}$ 


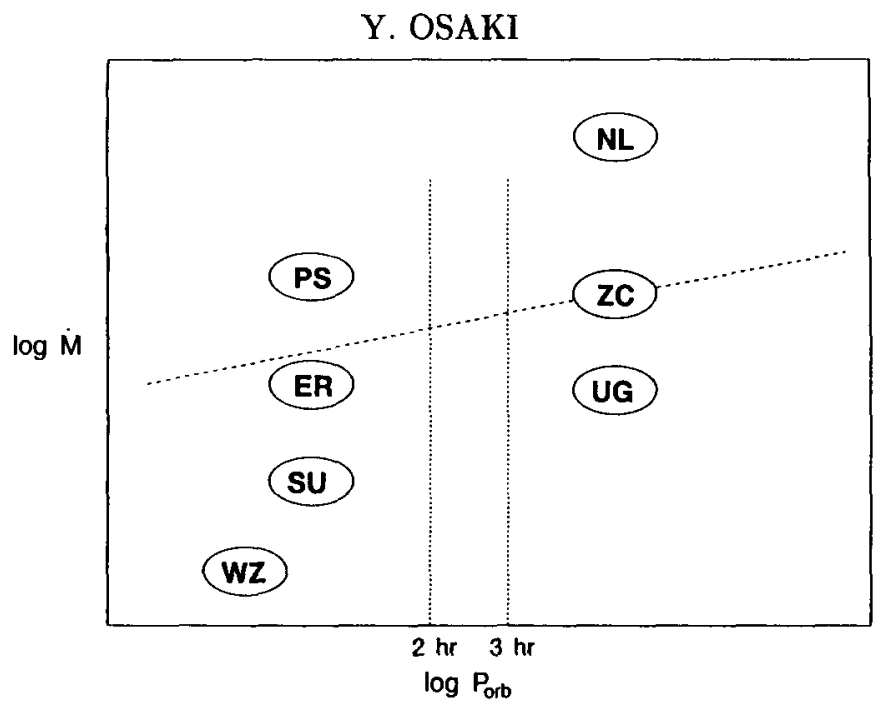

Figure 1. $P_{\text {orb }}-\dot{M}$ diagram showing different outburst behaviors of non-magnetic CVs. The region surrounded by dotted vertical lines shows the CV's $2 \ldots 3$ h period gap. The dashed line shows the border line between the thermally stable and unstable disks. Symbols in the figures are; NL: nova like stars, ZC: Z Cam stars, UG: U Gem stars, PS: 'permanent superhumpers', ER: ER UMa stars, SU: SU UMa stars, and WZ: WZ Sge stars. See text for more details.

are the masses of the mass-accreting primary star and the secondary star. The latter condition is in turn satisfied exclusively in cataclysmic variables (CVs) below the period gap.

The present author (Osaki 1989) has proposed the so-called 'thermaltidal instability model' to explain the superoutburst phenomenon of SU UMa stars, in which the two intrinsic instabilities, the thermal instability and tidal instability, are properly coupled. This model is basically within the general framework of the DI model.

An intriguing possibility has now opened in that almost all varieties of outburst light curves of dwarf novae may be explained by a single principle: the DI model. In this unification model, different outburst behaviors among non-magnetic CVs are basically classified by two parameters characterizing accretion disks in these systems; that is the orbital period of the system and mass transfer rate from the secondary star.

Fig. 1 schematically illustrates different classes of non-magnetic CVs in this unification model. They are basically divided into four regions depending on different combinations of the two intrinsic instabilities.

The CVs above the period gap can be classified into nova-like, Z Camtype DNe, and U Gem-type DNe depending on the thermal stability nature, which is in turn determined by the mass transfer rate. It has turned out that a similar classification of non-magnetic CVs below the period gap is possible. 


\section{Dwarf novae below the period gap}

Dwarf novae below the period gap are basically classified in the SU UMa sub-type that exhibit two distinct types of outbursts: more frequent, short 'normal outbursts' and less frequent, long 'superoutbursts'; a cycle from one superoutburst to the next one is called a 'supercycle'. The typical supercycle length is about a few hundred days.

Observations show a wide variety of activity within SU UMa stars. In fact, Vogt (1993) has classified SU UMa stars into three groups by their activity: group A, 'active' stars showing frequent outbursts (e.g. VW Hyi), group B, 'intermediate' in activity between two extremes (e.g. OY Car), and group C, very inactive stars or 'WZ Sge' stars that exhibit large outbursts on very rare occasions. Furthermore, a new type of extreme SU UMa stars called either 'ER UMa stars' or 'RZ LMi stars' has recently been discovered (Kato \& Kunjaya 1995; Robertson, Honeycutt \& Turner 1995), which exhibit extremely short supercycles of $19 \mathrm{~d}$ to $45 \mathrm{~d}$. We may thus add to the Vogt activity sequence two more groups: permanent superhumpers and ER UMa stars, and these two new groups should be put above Vogt's group A (see Fig. 1). Here permanent superhumpers are nova-like stars and thus thought to have hot thermally stable disks but still exhibit permanent superhumps (e.g. Skillman \& Patterson 1993).

We first discuss the case with $\dot{M}<10^{16} \mathrm{M}_{\odot} \mathrm{yr}^{-1}$. The present author (Osaki 1995a) has already suggested at the Garching conference and the Padova conference (Osaki 1995d) that Vogt's (1993) activity sequence may be explained as a sequence of decreasing mass transfer rate in the thermaltidal instability model. It is found in this model that the supercycle length, $T_{\mathrm{S}}$, is inversely proportional to mass transfer rate while the recurrence time, $T_{\mathrm{N}}$, of normal outbursts is inversely proportional to the square of mass transfer rate. Thus if the mass transfer rate is decreased along this sequence, the supercycle length is increased in proportion to the inverse of mass transfer rate while the number of normal outbursts in a supercycle decreases in proportion to the inverse of the supercycle length.

However, in order to explain the extremely long recurrence time of WZ Sge itself, $30 \mathrm{yr}$, it is not enough to decrease the mass transfer rate but also we need to decrease the viscosity parameter in the cold state, $\alpha_{\text {cold. }}$.

Let us now discuss the case of high mass transfer rate with $\dot{M}>$ $10^{16} \mathrm{M}_{\odot} \mathrm{yr}^{-1} \equiv \dot{M}_{16}$. As already noted, there is a critical mass transfer rate above which an accretion disk is always in a hot and thermally stable state. If the mass transfer rate is higher than the critical one, the system remains in a hot state. These CVs below the period gap are identified as permanent superhumpers, nova-like stars exhibiting permanent superhumps because 
they are thermally stable but tidally unstable.

Osaki $(1995$ b,c) has studied outburst light curves of the ER UMa stars. He has examined the supercycle length as a function of the mass transfer rate under fixed model parameters. It is found that the supercycle length is inversely proportional to the mass transfer rate if $\dot{M}_{16}<1$, as expected. However, if the mass transfer rate is further increased above $\dot{M}_{16} \sim 1$, the supercycle length has a broad minimum and it finally goes to infinity when the mass transfer rate becomes larger than the critical value for the thermal instability. ER UMa itself is found to be simulated very well as a system having a mass transfer rate corresponding to the broad minimum mentioned above (see Fig. 2 of Osaki 1995b).

For model parameters used to simulate ER UMa, the minimum supercycle length was about $45 \mathrm{~d}$ and thus a supercycle length as short as $19 \mathrm{~d}$ observed for RZ LMi could not be reproduced by these model parameters. It was found (Osaki 1995c) that the minimum supercycle length was sensitive to one model parameter that describes the strength of tidal torques during the supermaximum when the disk becomes eccentric. It has turned out that the supercycle length as short as $19 \mathrm{~d}$ of RZ LMi can be reproduced if the tidal torques during the superoutburst in RZ LMi are significantly weaker than those of ER UMa and ordinary SU UMa stars. The light curve of RZ LMi is also found to be simulated very well in the case of weaker tidal torques (see Fig. 2 of Osaki 1995c).

The wide variety in activity of CVs below the period gap indicates that the mass transfer rates from the secondary stars should have much wider range than that expected from the standard scenario for $\mathrm{CV}$ evolution based on gravitational-wave radiation. A cyclic variation in mass transfer rate in time scale of $10^{3} \ldots 10^{4} \mathrm{yr}$ is thus inferred.

\section{References}

Hirose, M., Osaki, Y., 1990, PASJ, 42, 135

Kato, T., Kunjaya, C., 1995, PASJ, 47, 163

Lubow, S.H., 1991, Ap. J., 381, 259

Osaki, Y., 1989, PASJ, 41, 1005

Osaki, Y., 1995a, PASJ, 47, 47

Osaki, Y., 1995b, PASJ, 47, L11

Osaki, Y., 1995c, PASJ, 47, L25

Osaki, Y. 1995d, in "Cataclysmic Variables", eds A. Bianchini et al., Kluwer, p307

Robertson, J.W., Honeycutt, R. K., Turner, G. W. 1995, PASP, 107, 443

Skillman, D.R., Patterson, J., 1993, Ap. J., 417, 298

Vogt, N., 1993, in "Cataclysmic Variables and Related Physics", eds O. Regev, G. Shaviv, Ann. Israel Phys. Soc., 10, 63

Whitehurst, R., 1988, MNRAS, 232, 35 